\title{
Optical Broadband Angular Selectivity
}

\author{
Yichen Shen,${ }^{1 \dagger}$ Dexin Ye,${ }^{3}$ Ivan Celanovic, ${ }^{1}$ Steven G Johnson,,${ }^{1,2}$ \\ John D Joannopoulos, ${ }^{1}$ and Marin Soljačić ${ }^{1}$ \\ ${ }^{1}$ Research Laboratory of Electronics, Massachusetts Institute of Technology, \\ Cambridge, MA 02139, USA \\ ${ }^{2}$ Department of Mathematics, Massachusetts Institute of Technology, \\ Cambridge, MA 02139, USA \\ ${ }^{3}$ Laboratory of Applied Research on Electromagnetics (ARE), Zhejiang University, \\ Hangzhou, 310027, China
}

†To whom correspondence should be addressed; E-mail: ycshen@mit.edu.

\begin{abstract}
:
Light selection based purely on the angle of propagation is a long-standing scientific challenge. In angularly selective systems, however, the transmission of light usually also depends on the light frequency. We tailored the overlap of the bandgaps of multiple one-dimensional photonic crystals, each with a different periodicity, in such a way as to preserve the characteristic Brewster modes across a broadband spectrum. We provide theory as well as experimental realization with an all-visible-spectrum, $p$-polarized angularly selective material system. Our method enables transparency throughout the visible spectrum at one angle, the generalized Brewster angle, and reflection at every other viewing angle.
\end{abstract}


The ability to control light has long been a major scientific and technological goal. In electromagnetic theory, a monochromatic electromagnetic plane wave is characterized (apart from its phase and amplitude) by three fundamental properties: its frequency, its polarization, and its propagation direction. The ability to select light according to each of these separate properties would be an essential step in achieving control over light (Fig. 1).

Tremendous progress has been made toward both frequency selectivity and polarization selectivity. Frequency selectivity (Fig. 1A) can be obtained, for example, by taking advantage of photonic bandgaps in photonic crystals (1-5). Polarization selectivity (Fig. 1B) is accomplished for example by means of a "wire grid" polarizer $(6)$ or by exploiting birefringent materials $(7,8)$. Methods based on interference and resonance effects have been explored for angular selectivity, but they have limited applications because they are sensitive to frequency.

An angularly selective material-system should ideally work over a broadband spectrum. Such a system could potentially play a crucial role in many applications, such as high efficiency solar energy conversion $(9,10)$, privacy protection $(11)$, and detectors with high signal-to-noise ratios. Some progress has been made towards achieving broadband angular selectivity by means of metallic extraordinary transmission $(12,13)$, anisotropic metamaterials $(14)$, combined use of polarizers and birefringent films (11), or geometrical optics at micrometer scale (15). The first two of these mechanism are difficult to realize in the optical regime; the other two work only as angularly selective absorbers.

Here, we introduce a basic principle to achieve optical broadband angular selectivity. Our result rests on i) the fact that polarized light transmits without any reflection at the Brewster angle, ii) the existence in photonic crystals of band gaps that prevent light propagation for given frequency ranges, and iii) the bandgap broadening effect of heterostructures. First, we prove our fundamental idea theoretically for a single polarization and oblique incident angles, and also for both polarizations and normal angle of incidence. Second, we experimentally demonstrate the concept in the case of all-visible spectrum, $p$-polarized light. The demonstrator is transparent 
for all colors at one viewing angle and highly reflecting at every other viewing angle.

We begin by considering a simple quarter-wave stack with periodicity $a$, relative permeability $\mu$ of $\mu_{1}=\mu_{2}=1$, and relative permittivities $\epsilon$ of $\epsilon_{1}$ and $\epsilon_{2}$. In such a system, monochromatic plane waves with frequency $\omega$ propagate only in certain directions; propagation in other directions is not allowed because of destructive interference (3). Another way to look at this is through the photonic band diagram shown in Fig. 2A: Modes that are allowed to propagate (so-called extended modes) exist in the shaded region; no modes are allowed to propagate in the white regions (known as bandgaps). In the photonic band diagram, modes with propagation direction forming an angle $\theta_{i}$ with respect to $\hat{z}$ axis in Fig. 2 (in the layers with dielectric constant $\epsilon_{i}$ ) lie on a straight line represented by $\omega=k_{y} c /\left(\sqrt{\epsilon_{i}} \sin \theta_{i}\right)$, where $k_{y}$ is the $\hat{y}$ component (as defined in Fig. 2) of the wave vector $\vec{k}$ and $c$ is the speed of light; for general propagation angle $\theta_{i}$, this line will extend through the regions of the extended modes, as well as through the bandgap regions.

However, for $p$-polarized light, there is a special propagation angle, known as the Brewster angle $\theta_{B}$, for which the extended modes exist regardless of $\omega$ (dashed line in Fig. 2A) $(8,16)$.

$$
\theta_{B}=\tan ^{-1} \sqrt{\frac{\epsilon_{2}}{\epsilon_{1}}}
$$

where $\theta_{B}$ is the Brewster angle the layers with dielectric constant $\epsilon_{1}$. At $\theta_{B}, p$-polarized light is fully transmitted for all frequencies at both interfaces (from $\epsilon_{1}$ to $\epsilon_{2}$ layers and from $\epsilon_{2}$ to $\epsilon_{1}$ layers). This condition is not sufficient to achieve angular selectivity; we also need to remove all the extended modes in other propagation directions. Because the location of the bandgap scales proportionally to the periodicity of the quarter-wave stack, the effective bandgap can be enlarged when we stack quarter-wave stacks with various periodicities together (17-19). The details of this process are illustrated in fig. S1 (20). As a proof of principle, in Fig. 2D we plot the band diagram of an ideal structure with $\epsilon_{1}=1$ and $\epsilon_{2}=2$ and the number of quarter-wave stacks approaching infinity. By doing this with a finite system of 50 stacks (10 bi-layers in each stack), we can achieve an angularly selective range of less than $2^{\circ}$ and a frequency bandwidth 
of $\geq 54 \%$, similar to the size of the visible spectrum (Fig. 2G).

For $s$-polarized light, as there is no Brewster angle, this construction behaves as a dielectric mirror that reflects over a wide frequency range and over all incident angles (Fig. S2) (20).

The mechanism above provides both angular selectivity and polarization selectivity, and is therefore useful in many applications. For example, in most optically pumped lasers, the pumping light comes in with a specific polarization and at one specific angle. A cavity built with both angularly selective and polarization- selective mirrors will allow the pumping light to get through, while at the same time trapping all the light with other propagation directions and polarizations inside the cavity.

The restriction on the polarization can be lifted by releasing the conventional requirement that $\mu_{1}=\mu_{2}=1$. During the past decade, it has been demonstrated that metamaterials have the potential to achieve $\epsilon=\mu \neq 1$ in broad frequency range (21-23). Consider two media with $\epsilon_{1}=\mu_{1} \neq \epsilon_{2}=\mu_{2}$; under those circumstances there is no reflection at the interface at normal incidence because the two media are impedance matched, where the impedance $Z$ is defined as $Z=\sqrt{\frac{\underline{\mu}}{\epsilon}}$. The off-axis reflectivity can be calculated directly from the generalized Fresnel equations (8):

$$
\left(\frac{E_{r}}{E_{i}}\right)_{\perp}=\frac{\frac{1}{Z_{i}} \cos \theta_{i}-\frac{1}{Z_{t}} \cos \theta_{t}}{\frac{1}{Z_{i}} \cos \theta_{i}+\frac{1}{Z_{t}} \cos \theta_{t}}
$$

and

$$
\left(\frac{E_{r}}{E_{i}}\right)_{\|}=\frac{\frac{1}{Z_{t}} \cos \theta_{i}-\frac{1}{Z_{i}} \cos \theta_{t}}{\frac{1}{Z_{i}} \cos \theta_{i}+\frac{1}{Z_{t}} \cos \theta_{t}},
$$

where the subscripts $i$ and $r$ denote incident light and reflected light, respectively, and the subscripts $\perp$ and $\|$ indicate the direction of the electric field $\vec{E}$ with respect to the plane of incidence. When $Z_{i}=Z_{t}$, the reflectivities for $s$ - and $p$ - polarized light become identical. In particular, the Brewster angle is the same for both polarizations $\left(\theta_{B}=\theta_{i}=\theta_{r}=0^{\circ}\right)$. As a proof of principle we plot the band diagram of a quarter-wave stack with $\epsilon_{1}=\mu_{1}=1$, and $\epsilon_{2}=\mu_{2}=2$ in Fig. 2 C. As in the previous case, we can broaden the bandgaps by stacking 
quarter-wave stacks with various periodicities together (Fig. 2F); this approach gives rise to ultra-broadband angular selectivity at normal incidence for both polarizations (Fig. 2H).

Other than using photonic bandgaps to remove unwanted extended modes, there might exist even more optimized ways to forbid light from propagating in unwanted directions: The narrowness of angular selectivity can be optimized using numerical tools $(17,24)$ to further enhance the performance of the material system. Examples of optimizations based on three different physical mechanisms are shown in fig. S3 (20).

To show the feasibility of the method described above, we present an experimental realization for the $\epsilon_{1} \neq \epsilon_{2}, \mu_{1}=\mu_{2}=1$ case. The sample was fabricated with the bias target deposition (BTD) technique (25) at 4Wave Inc, using $\mathrm{SiO}_{2}\left(\epsilon_{1} \approx 2.18, \mu_{1}=1\right)$ and $\mathrm{Ta}_{2} \mathrm{O}_{5}$ $\left(\epsilon_{2} \approx 4.33, \mu_{2}=1\right.$ ) on a $2 \mathrm{~cm} \times 4 \mathrm{~cm}$ fused silica wafer (University Wafer Inc.). The sample consists of 84 layers in total (Fig. 2E). There are six bi-layer stacks $(m=6)$, each bi-layer stack consisting of seven bi-layers $(n=7)$, with the thicknesses of each layer equal in a given stack. The periodicities of the six bi-layer stacks form a geometric series with $a_{i}=a_{0} r^{i-1}$, for the $\mathrm{i}^{\text {th }}$ stack, where $a_{0}=140 \mathrm{~nm}$ and $r=1.165$. For index matching purposes, the whole sample was immersed into a colorless liquid with dielectric constant $\epsilon_{\text {liquid }}=\epsilon_{1}=2.18$ (Cargille Labs) (Fig. 3A). The sample could work in the air by adding a coupling prism or by using a porous material for $\epsilon_{1}$ that has a lower refractive index, such as aerogel (26).

The sample is transparent (up to 98\%) to $p$-polarized incident light at $\theta_{B}=55^{\circ}$ (Fig. 3D); the angular window of transparency is about $8^{\circ}$. It behaves like a mirror at all other incident angles over the entire visible spectrum (Fig. 3B,C,E). For $s$-polarized incident light, the sample behaves like a mirror at all angles (fig. S2) (20). The p-polarization transmittance of the sample in the visible spectrum was measured using an ultraviolet-visible spectrophotometer (Cary500i); a $p$-polarizer was used to filter the source beam. The experimentally measured result agrees with the rigorous coupled wave analysis (RCWA) (27) simulation prediction (Fig. 4), which includes the measured dispersion of materials (index variation $<1.3 \%$ for $\mathrm{SiO}_{2}$ and $<6.2 \%$ for $\mathrm{Ta}_{2} \mathrm{O}_{5}$ 
over the wavelength range from 400 to $700 \mathrm{~nm}$ ). In the experimental measurements, the peak transmittance at $\theta_{B}$ becomes lower at shorter wavelengths (Fig. 4B) because the wavelength is getting closer to the dimensional tolerance of fabrication.

Movie $\mathrm{S} 1$ is a video recording of the full process with the sample rotating $90^{\circ}$ in this experimental setup is provided in the supplementary materials (20).

Our method has a number of attractive features, including simplicity, narrow angle selectivity, scalability beyond optical frequencies, and reproducibility on large scales. Furthermore, this method can be implemented in other wave systems that have Brewster angle analogs, such as acoustic and elastic waves. A natural next step would be to examine materials whose magnetic permeability is similar to their dielectric constant, so as to reach angular selectivity in both polarizations.

\section{References and Notes}

1. E. Yablonovitch, Phys. Rev. Lett. 58, 2059 (1987).

2. E. Yablonovitch, J. Opt. Soc. Am. B 10, 283 (1993).

3. J. Joannopoulos, S. Johnson, J. Winn, R. Meade, Photonic Crystals: Molding the Flow of Light (Second Edition) (Princeton University Press, 2011).

4. S. G. Johnson, J. D. Joannopoulos, Appl. Phys. Lett. 77, 3490 (2000).

5. Y. Fink, et al., Science 282, 1679 (1998).

6. S. Sriram, K. Steinbruegge, E. Supertzi. US patent 4512638 filed 31 Aug 1982, and issued 23 Apr 1985.

7. J. F. Archard, A. M. Taylor, J. Sci. Instrum. 25, 407 (1948).

8. E. Hecht, Optics (Pearson Education, 2008). 
9. O. Hohn, et al., Proc. SPIE 8438, 84380A (2012).

10. P. Bermel, et al., Opt. Express 18, A314 (2010).

11. S. W. MacMaster. US patent 7052746 filed 26 Nov 2003, and issued 30 May 2006.

12. A. Alù, G. D’Aguanno, N. Mattiucci, M. J. Bloemer, Phys. Rev. Lett. 106, 123902 (2011).

13. N. Aközbek, et al., Phys. Rev. B 85, 205430 (2012).

14. R. E. Hamam, I. Celanovic, M. Soljačić, Phys. Rev. A 83, 035806 (2011).

15. E. D. Kosten, J. H. Atwater, J. Parsons, A. Polman, H. A. Atwater, Light Sci Appl 2 (2013).

16. J. N. Winn, Y. Fink, S. Fan, J. D. Joannopoulos, Opt. Lett. 23, 1573 (1998).

17. B. Perilloux, Thin-film Design: Modulated Thickness and Other Stopband Design Methods, Tutorial texts in optical engineering (SPIE Press, 2002).

18. C. Zhang, F. Qiao, J. Wan, J. Zi, J. Appl. Phys. 87, 3174 (2000).

19. X. Wang, et al., Appl. Phys. Lett. 80, 4291 (2002).

20. Supporting Materials available on Science online.

21. J. B. Pendry, A. J. Holden, D. J. Robbins, W. J. Stewart, IEEE Trans. Microw. Theory Techn. 47, 2075 (1999).

22. J. B. Pendry, A. J. Holden, W. J. Stewart, I. Youngs, Phys. Rev. Lett. 76, 4773 (1996).

23. J. Shin, J.-T. Shen, S. Fan, Phys. Rev. Lett. 102, 093903 (2009).

24. J. Xu, J. Lightwave Technol. 28, 1114 (2010).

25. V. V. Zhurin, H. Kaufman, J. R. Kahn, T. L. Hylton, J. Vac. Sci. Technol., A 18, 37 (2000).

26. D. W. Schaefer, K. D. Keefer, Phys. Rev. Lett. 56, 2199 (1986). 
27. V. Liu, S. Fan, Comput. Phys. Commun. 183, 2233 (2012).

28. S. G. Johnson. The NLopt nonlinear-optimization package, http://ab-initio.mit.edu/nlopt.

Acknowledgments: We thank Paola Rebusco for critical reading and editing of the manuscript, Jay J Senkevich for the advice on fabricating the sample, and Chia-Wei Hsu for the valuable discussion. This work was partially supported by the Army Research Office through the ISN under Contract Nos. W911NF-13-D0001. The fabrication part of the effort, as well as (partially) M.S. were supported by the MIT S3TEC Energy Research Frontier Center of the Department of Energy under Grant No. DE-SC0001299. 

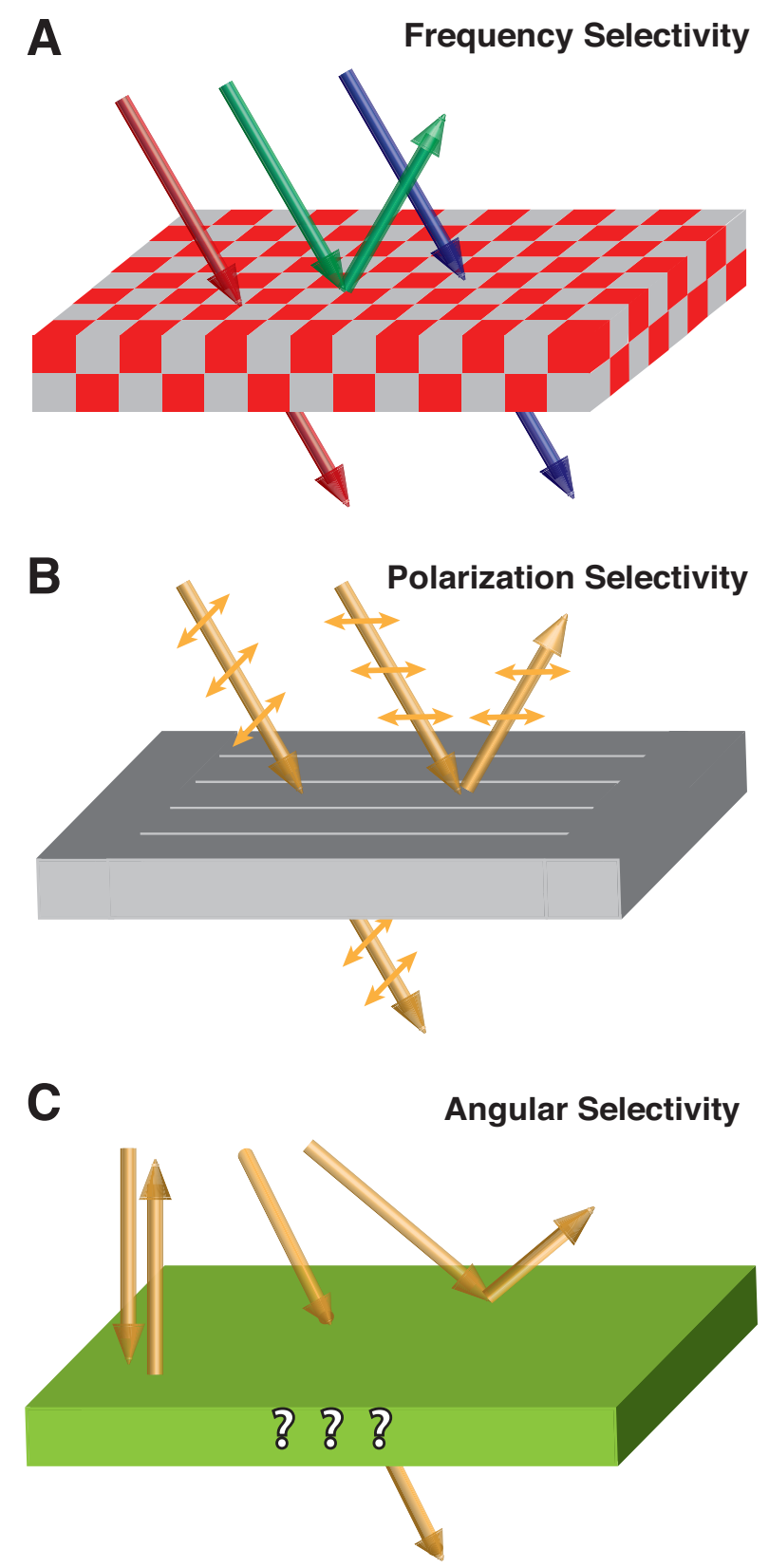

Fig. 1: Illustration of light selection on the basis of its fundamental properties (A) Frequency selectivity provides control over transmission/reflection of different frequencies. Photonic crystals (such as omnidirectional mirrors) can select light in specific frequency bandwidths. (B) Polarization selectivity provides control over the transmission/reflection of different polarizations. An ideal polarizer selects light with a specific polarization. (C) Angular selectivity provides control over the transmission/reflection of incident angles; so far achieving broadband selectivity has remained illusive. 
A

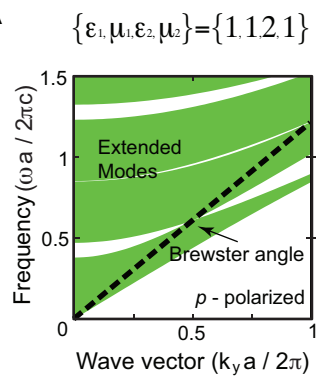

D

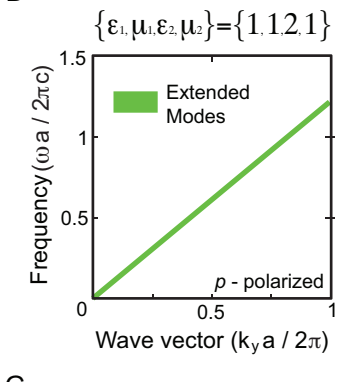

G

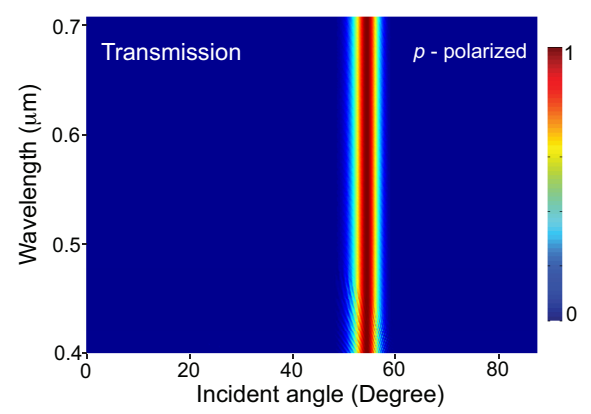

E
C
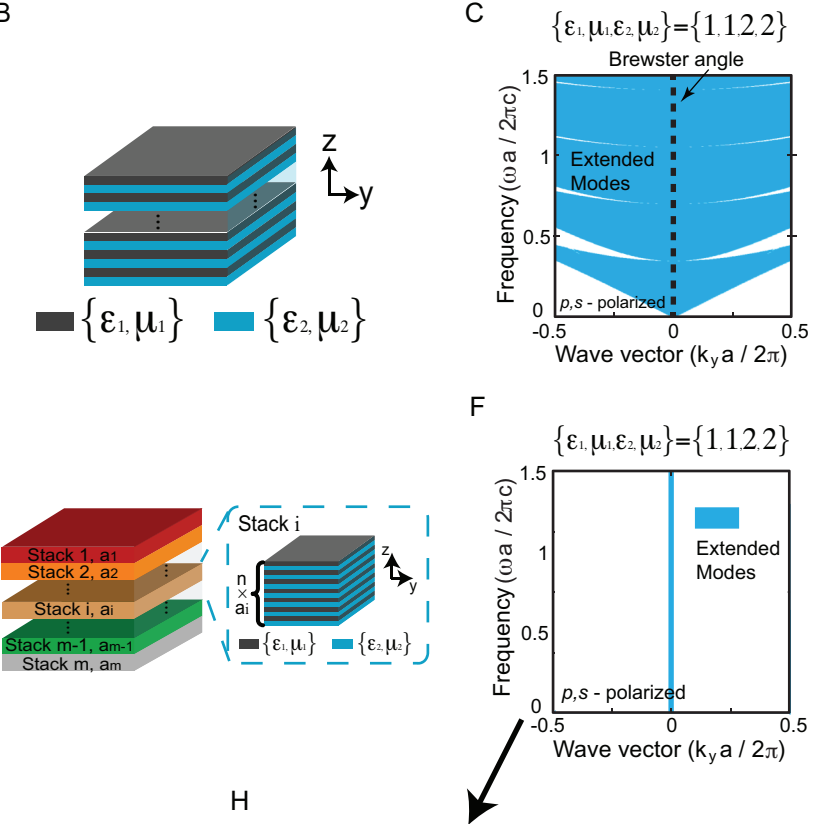

$\mathrm{H}$

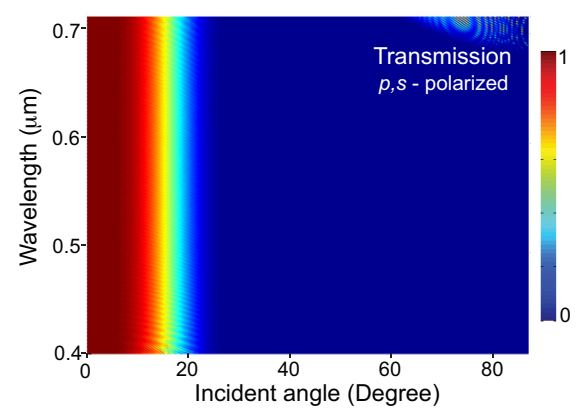

Fig. 2: Theoretical illustration. (A) Extended modes (shaded regions) for off-axis propagation vectors $\left(0, k_{y}, k_{z}\right)$ in a quarter-wave stack with two materials having $\epsilon_{1}=1$ and $\epsilon_{2}=2$ respectively. The green region indicates modes with $\mathbf{E}$ fields polarized in the $y z$ incidence plane (TE or $p$-polarized). The dashed black line corresponds to the Brewster angle in both layers. (B) Schematic layout of a simple quarter-wave stack. (C) The same plot as in part (A), but with $\epsilon_{1}=\mu_{1}=1, \epsilon_{2}=\mu_{2}=2$, and for both polarizations. (D,F) Extended modes for an ideal heterostructure with $(m, n) \rightarrow \infty$. (E) Schematic layout of the heterostructure stacking mechanism $(\mathrm{G}) p$-polarized transmission spectrum of 50 stacks of quarter-wave stacks at various periodicities. Each quarter-wave stack consists of 10 bi-layers of $\left\{\epsilon_{1}=1, \epsilon_{2}=2\right\}$ materials. The periodicities of these quarter-wave stacks form a geometric series $a_{i}=a_{0} r^{i-1}$ with $a_{0}=200 \mathrm{~nm}$ and $r=1.0212$, where $a_{i}$ is the periodicity of $i^{t h}$ stack. A more detailed discussion on how this stacking process works is presented in the supplementary material. $(\mathrm{H})$ $p, s$-polarized transmission spectrum for a structure that has the same number of stacks and layers per stack as in part (G), but with $a_{0}=140 \mathrm{~nm}$ and $r=1.0164$, and with different material properties: $\epsilon_{1}=\mu_{1}=1, \epsilon_{2}=\mu_{2}=2$. 
A Top Vlew:

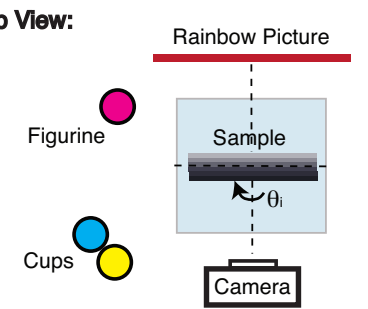

B

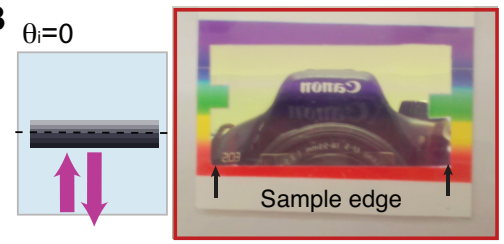

D

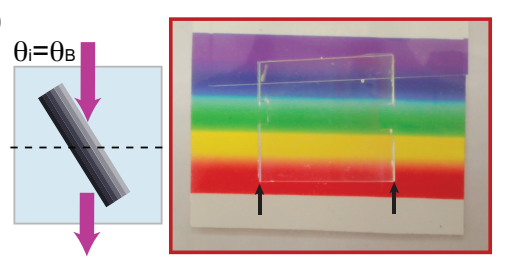

Side View:
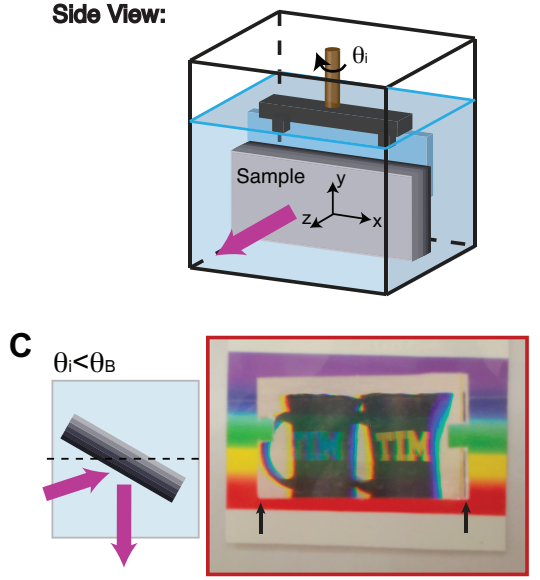

$E$

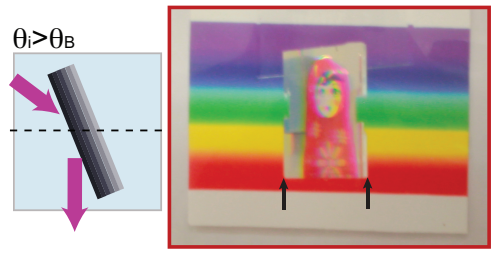

Fig. 3: Experimental setup and observation. (A) Schematic layout of the experimental setup. The system is immersed in a liquid that is index matched to $\epsilon_{1}=\epsilon_{\mathrm{SiO}_{2}}=2.18$. (B)Normal incident angle setup. The sample behaves as a mirror and reflects the image of the camera. (C) $\theta_{i}=30^{\circ}$ setup. The sample behaves as a mirror and reflects the image of MIT cups in the lab. (D) $\theta_{i}=\theta_{B}=55^{\circ}$ setup. The sample becomes transparent for the entire visible regime for $p$-polarized light. (E) $\theta_{i}=70^{\circ}$ setup. The sample behaves as a mirror and reflects the figurine placed at the corner of the table. In B-E a polarizer is installed on the camera so it detects only $p$-polarized light. 

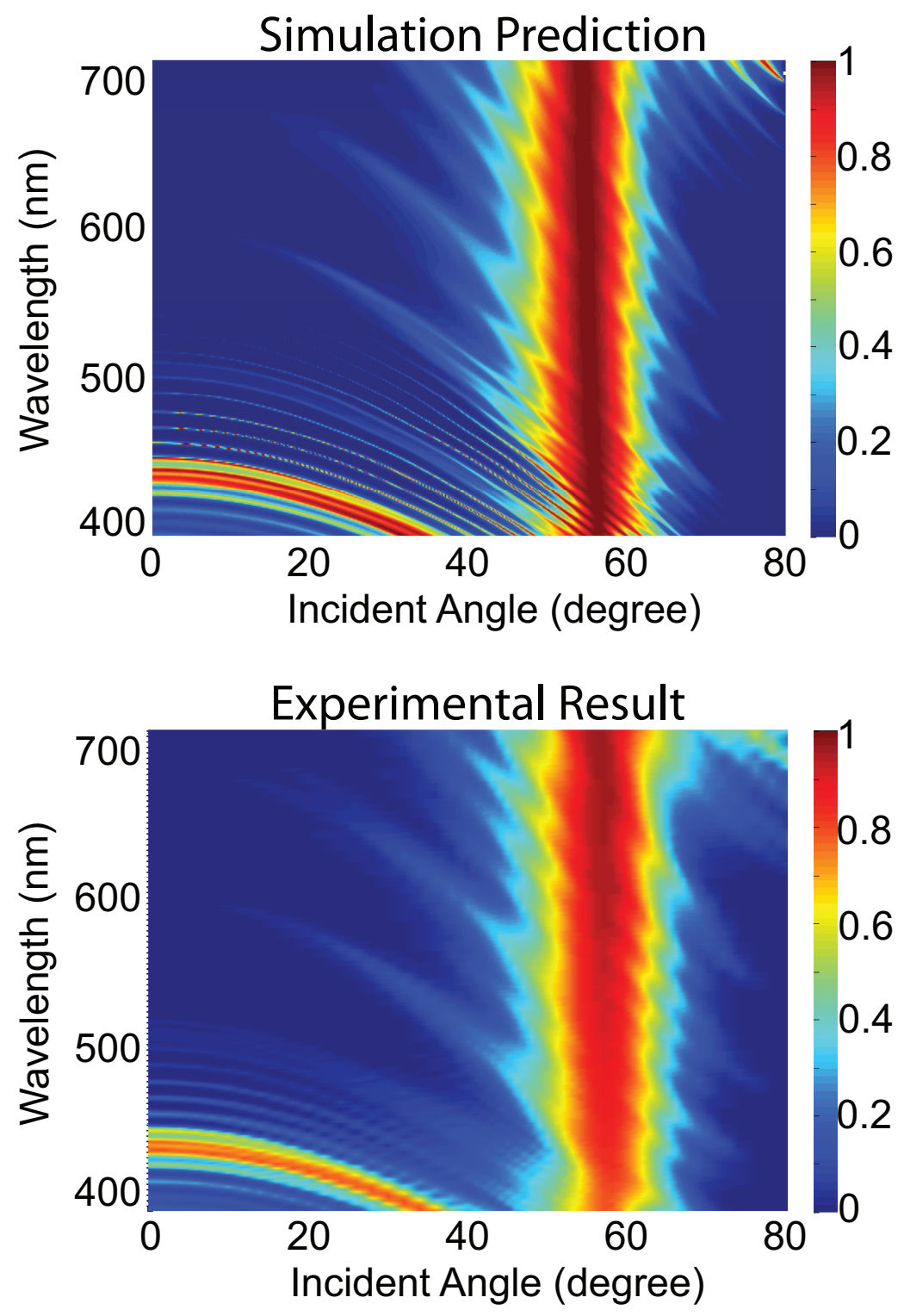

Fig. 4: Simulation and experimental measurement. Comparison between the $p$-polarized transmission spectrum of the Rigorous Coupled Wave Analysis (RCWA) simulation (27) and the corresponding experimental measurements. 


\section{Supplementary Materials}

www.sciencemag.org

Figs. S1, S2, S3

Reference (28)

Movie S1 


\section{Supplementary Materials for}

\section{Optical Broadband Angular Selectivity}

Yichen Shen, ${ }^{*}$ Dexin Ye, Ivan Celanovic, Steven G Johnson, John D Joannopoulos, and Marin Soljačić

*To whom correspondence should be addressed; E-mail: ycshen@mit.edu. 
A
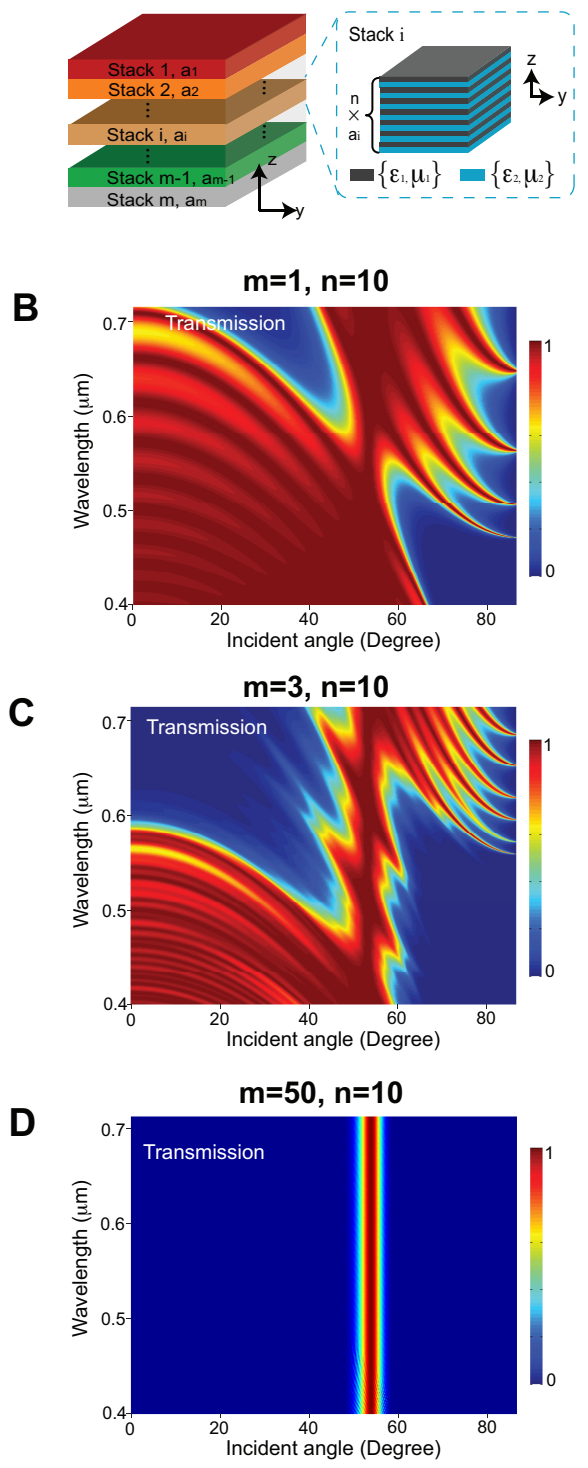

Fig. S1: Effect of Photonic Crystal Stacking for $p$-polarized light (A) Schematic illustration of the stacking mechanism. The dielectric constant of two materials are chosen to be $\epsilon_{1}=1$ and $\epsilon_{2}=2$ (B) Transmission spectrum of a simple quarter-wave stack with 10 bi-layers $(1$ stack), a=340 nm. (C) Transmission spectrum of 3 stacks of 10 bi-layer quarter-wave stacks, $a_{1}=280 \mathrm{~nm}, a_{2}=335 \mathrm{~nm}, a_{3}=400$ nm. (D) Transmission spectrum of 50 stacks of 10 bi-layer quarter-wave stacks. It is the same plot as in Fig. 2(G). 
A

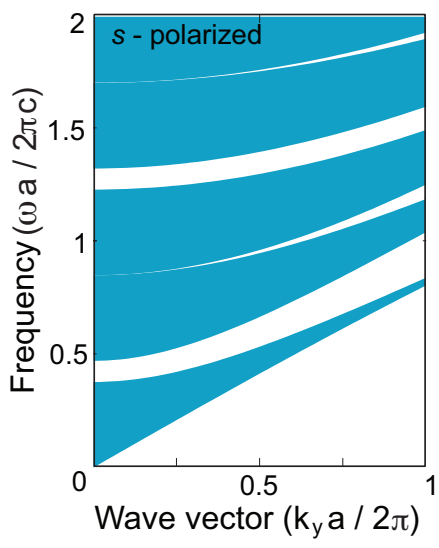

C

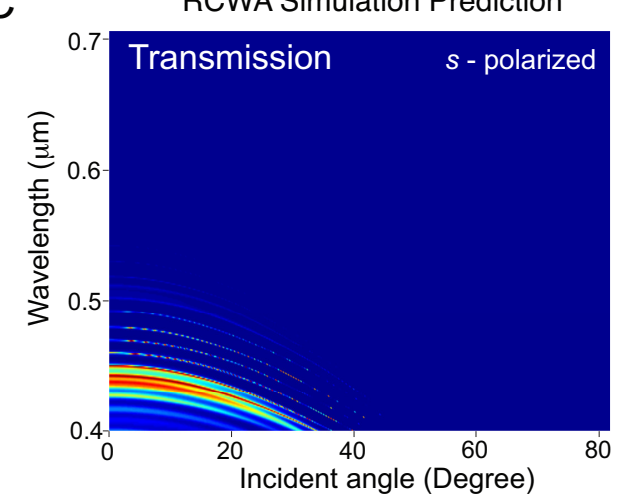

B Transfer Matrix Optimization for 1000 layers

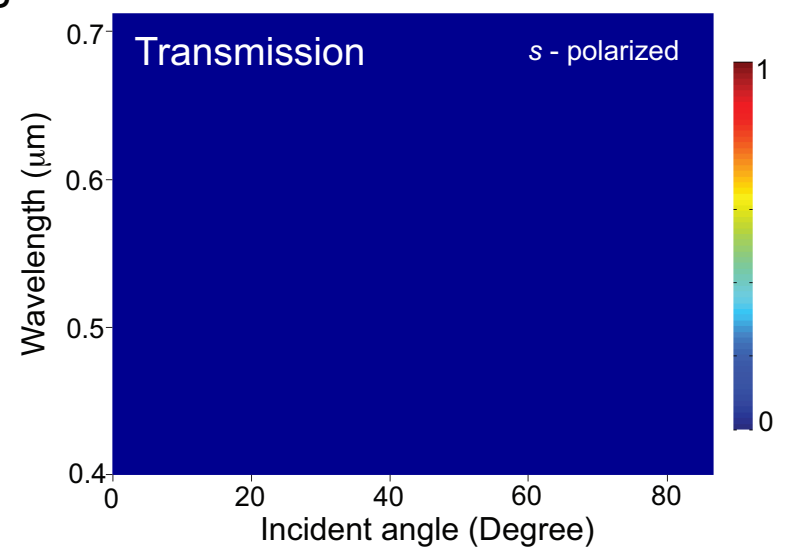

D

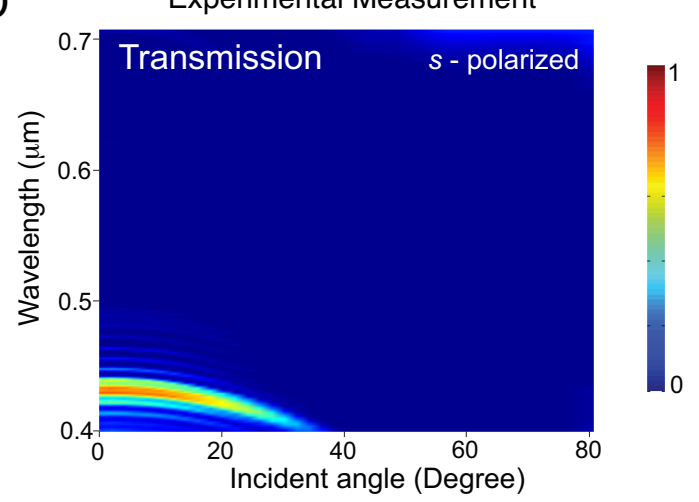

Fig. S2: $s$-polarization discussion (A) $s$-polarized extended modes (shaded regions) for off-axis propagation vectors $\left(0, k_{x}, k_{z}\right)$ in a quarter-wave stack with two materials having $\epsilon_{1}=1$ and $\epsilon_{2}=2$ respectively. It is the same plot as Figure $2(\mathrm{~A})$ in the main text, but this time for $s$-polarization. (B) $s$-polarized transmission spectrum for the same optimized structure described in Figure 2(G). (C,D) s-polarization analogues of Fig. 4 in the main text. 
A

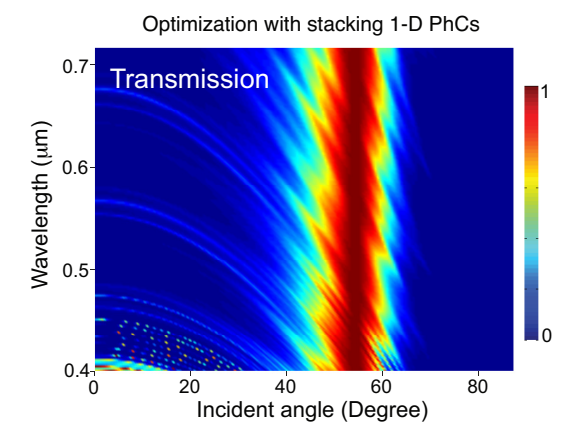

B
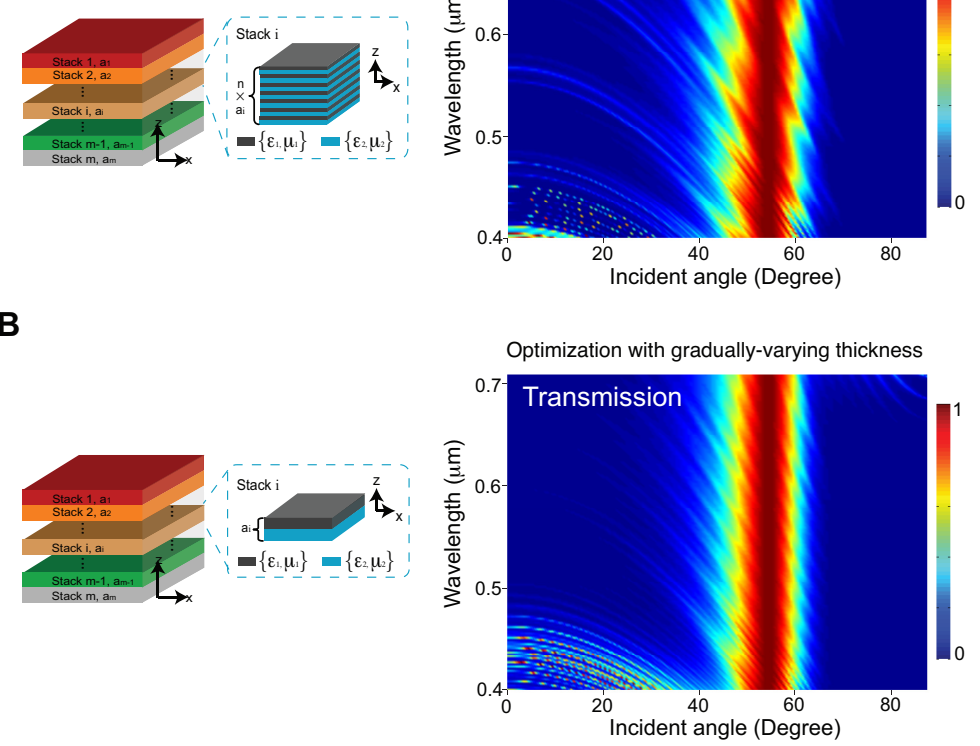

C

Optimization by setting each layer thickness as a free parameter
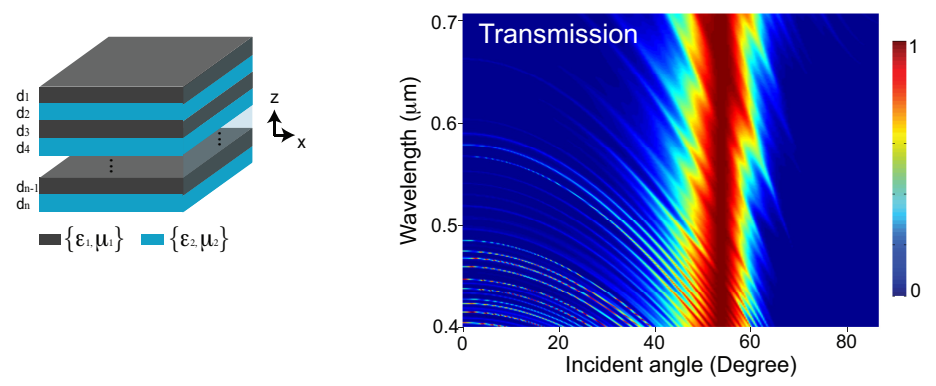

Fig. S3: Optimization of the Narrowness of Angular Selectivity We choose the figure of merit (FOM) of our optimization to be the minimum of the total transmitted power over all the angles in the visible regime. Given the dielectric constant of the materials we use $\left(\epsilon_{1}\right.$ and $\left.\epsilon_{2}\right)$, incoming light at the Brewster's angle always has $100 \%$ transmission. Therefore, a smaller total transmitted power corresponds to a narrower window of angular transparency. For each of the three optimizations below, we set the total number of layers to be 100 , and the material properties to be $\left\{\epsilon_{1}, \mu_{1}, \epsilon_{2}, \mu_{2}\right\}=\{1,1,2,1\}$.(A) Transmission spectrum of $p$-polarized light for the structure optimized based on stacking 1-D photonic crystals (as illustrated in Supplementary Fig. 1); here we fixed $\mathrm{n}=10$, and the optimized periodicities are $\left\{a_{1}, a_{2}, a_{3}, a_{4}, a_{5}\right\}=\{200,259,335,433,560\} \mathrm{nm}$. (B) Transmission spectrum for the structure optimized based on geometrically varying the thickness of each bi-layer $(n=1$ case in part $(\mathrm{A}))$. The thickness of each bi-layer forms a geometric series $a_{i}=a_{0} r^{i-1}$ with the optimized $a_{0}=200 \mathrm{~nm}$ and $\mathrm{r}=1.0212$. the total transmitted power in this optimization is $3 \%$ better than the result in part (A). (C) Transmission spectrum for the structure optimized based on Constrained Optimization BY Linear Approximations (COBYLA) algorithm (27). We set each layer thickness as a free parameter; the initial condition is set to the result obtained in part (B) above. The total transmitted power in this optimization is $10 \%$ better than the result in part $(\mathrm{A})$ 


\section{References and Notes}

27. Steven G. Johnson, The NLopt nonlinear-optimization package, http://ab-initio.mit.edu/nlopt 